\title{
A Kalman filtering approach to particle track filtering and track uncertainty quantification for 3D PTV measurements
}

\author{
R. Sethu Viji, J. Eshraghi, J. Zhang, M. C. Brindise, S. Bhattacharya, \\ P. P. Vlachos* \\ Purdue University, Department of Mechanical Engineering, West Lafayette, IN 47907, USA \\ *pvlachos@purdue.edu
}

\begin{abstract}
Three-dimensional Particle Tracking Velocimetry (3D-PTV) is a non-invasive flow measurement technique that computes the velocity field by reconstructing 3D particle positions of individual tracer particles and by subsequently tracking those positions. The particle velocity measurement accuracy depends on the faithful reconstruction of 3D particle positions. The complex measurement chain in 3D-PTV involves several steps, from calibration to 3D position reconstruction and particle position tracking, each having its own source of error. Additionally, higher seeding density increases the uncertainty in particle reconstruction and tracking, which in turn, increases the noise in the estimated tracks. A noisy track decreases the measurement accuracy and amplifies any noise in the PTV-derived quantities of interest, which includes acceleration, pressure and vorticity. Thus, track filtering techniques are critical in a 3D-PTV measurement. Track fitting using polynomial functions, filtering methods adopted from signal processing and object tracking are among the well-established techniques used to achieve smooth position, velocity estimates from reconstructed particle trajectories. The Kalman filter is one such filtering technique that is widely used in various applications. The strength of the Kalman filter lies in its ability to perform noise reduction that is informed by existing physical models and the uncertainty estimates of recorded measurements. However, the measurement uncertainty input to the Kalman filter needs to be known at priori, which in many cases may not be available or could be difficult to estimate. In the literature on Kalman filters and their variants applied to 2D-PIV/PTV, the position uncertainty data fed to the filter is either user-defined or estimated based on global noise levels in the PTV measurements. But instantaneous position and velocity uncertainty quantification for individual particle positions/tracks has been challenging in the 3D PTV community. Recent work by Bhattacharya and Vlachos (2020) provides an estimate of the uncertainty in the reconstructed particle positions for a 3D PTV measurement. This position uncertainty estimate dynamically updates the filter gain for each track and enables the evaluation of the performance of the Kalman filter in 3D PTV track filtering.
\end{abstract}

The Kalman Filter $(\mathrm{KF})$ reconstructs smooth tracks from noisy experimental particle trajectories through a series of predictor-corrector steps. Taking advantage of physical models that describe Lagrangian particle tracking, KF internally evaluates the model prediction of a particle's current state $\left(X_{p}\right)$. This is then fused with the recorded experimental data $\left(Y_{p}\right)$ at the current frame to correct the prediction. The weighting applied on the two pieces of information, the model predictions and experimental data, towards the final estimate depends on the relative uncertainty associated with each experimental data point $(R)$ and model prediction $(P)$. This relative uncertainty is represented by the Kalman Gain factor $(K G)$, defined as:

$$
K G=\frac{P_{p}}{P_{p}+R}
$$

for one-dimensional KF, also referred to as P-type KF filter (Yagoh et al. (1992)). Here $P_{p}$ corresponds to the uncertainty propagated through the predictor step. The filtered position estimate is computed as:

$$
X_{f}=K G \cdot Y_{p}+(1-K G) \cdot X_{p}
$$




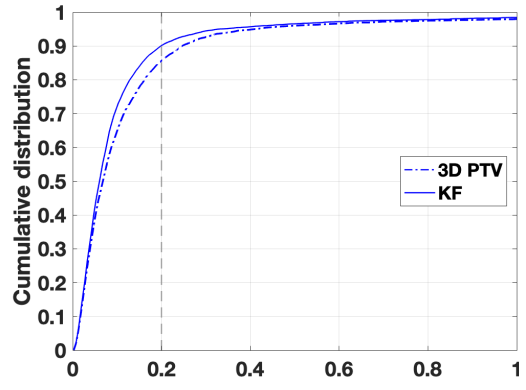

(a) Noise $0 \%$

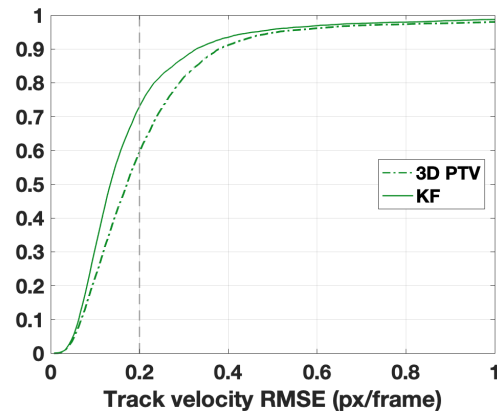

(b) Noise $5 \%$

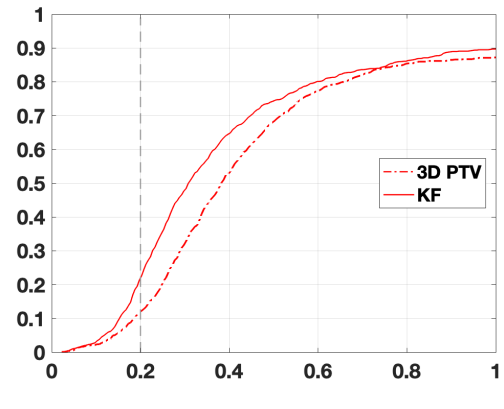

(c) Noise $10 \%$

Figure 1: Cumulative distribution of RMS error in track velocity estimates for a 3D synthetic vortex ring, generated at a seeding density of $0.025 \mathrm{ppp}$. The improvement in KF filtered result is reported at 0.2 pixels/frame.

Equation 2 also represents the input to the physical model in the next iteration. Hence, a given particle track measurement is reconstructed iteratively by taking into consideration the instantaneous position uncertainties associated with the experimental data. The filtered tracks are bound by a reduced set of position uncertainty values determined by KF.

The investigation on the performance of the P-type KF applied to the 3D-PTV position and velocity measurements is twofold: one on the tracked particle positions and the other on particle velocity estimates. The performance assessment is based on a synthetic 3D vortex ring (Wu et al.(2006)). More focus is directed towards analyzing the track velocity estimates, considering the wide dynamic range in the dataset chosen. For the velocity comparison, the filtered position estimates from KF are converted to particle velocities by applying a second-order central difference scheme, while the PTV velocity estimates are obtained from 3D-PTV nearest neighbor. The current results for the noisy synthetic data (Fig.1) show more than 20\% improvement in track velocity error compared to regular 3D PTV estimation when uncertainty informed Kalman filtering is used. Additionally, a Gaussian Smoothing (GS) filter can be used to compute velocities from the KF position estimates. The KF estimated track uncertainty would inform the GS on the level of velocity smoothing required. Thus, the proposed Kalman filtering approach provides an improved estimate of the particle trajectory from a noisy regular 3D PTV track and also quantifies the uncertainty in the filtered track. The current framework is further tested and validated for an experimental pipe flow case. The obtained results would be compared against velocity tracks estimated from FlowFit (Gesemann et al. (2016)).

\section{References}

Bhattacharya S and Vlachos PP (2020) Volumetric particle tracking velocimetry (PTV) uncertainty quantification. Experiments in Fluids 61:1-18

Gesemann S, Huhn F, Schanz D, and Schröder A (2016) From noisy particle tracks to velocity, acceleration and pressure fields using B-splines and penalties. in 18th international symposium on applications of laser and imaging techniques to fluid mechanics, Lisbon, Portugal (pp. 4-7).

Wu JZ, Ma HY, and Zhou MD (2006) Vorticity and Vortex Dynamics. pages 271-275. Springer, Berlin, Heidelberg

Yagoh K, Ogawara K, and Iida SI (1992) The particle tracking method using the kalman filter. in Flow Visualization VI (pp. 838-842). Springer, Berlin, Heidelberg 